ARTICLE

https://doi.org/10.1038/s41467-019-11826-1

\title{
Distinct methylation levels of mature microRNAs in gastrointestinal cancers
}

\author{
Masamitsu Konno (1) 1,10, Jun Koseki2,10, Ayumu Asai1,2,10, Akira Yamagata ${ }^{3,10, ~ T e p p e i ~ S h i m a m u r a 4, ~}$ \\ Daisuke Motooka $^{5}$, Daisuke Okuzaki (ib ${ }^{5}$, Koichi Kawamoto ${ }^{6}$, Tsunekazu Mizushima ${ }^{6}$, Hidetoshi Eguchi ${ }^{6}$, \\ Shuji Takiguchi ${ }^{6,7}$, Taroh Satoh ${ }^{1}$, Koshi Mimori $^{8}$, Takahiro Ochiya ${ }^{9}$, Yuichiro Doki ${ }^{6}$, Ken Ofusa ${ }^{3}$, Masaki Mori ${ }^{6}$ \& \\ Hideshi Ishii ${ }^{2}$
}

The biological significance of micro (mi)RNAs has traditionally been evaluated according to their RNA expression levels based on the assumption that miRNAs recognize and regulate their targets in an unvarying fashion. Here we show that a fraction of mature miRNAs including miR-17-5p, -21-5p, and -200c-3p and let-7a-5p harbor methyl marks that potentially alter their stability and target recognition. Importantly, methylation of these miRNAs was significantly increased in cancer tissues as compared to paired normal tissues. Furthermore, miR-17-5p methylation level in serum samples distinguished early pancreatic cancer patients from healthy controls with extremely high sensitivity and specificity. These findings provide a basis for diagnostic strategies for early-stage cancer and add a dimension to our understanding of miRNA biology.

\footnotetext{
${ }^{1}$ Department of Frontier Science for Cancer and Chemotherapy, Graduate School of Medicine, Osaka University, Suita, Osaka, Japan. ${ }^{2}$ Department of Cancer Profiling Discovery/ Medical Data Science, Graduate School of Medicine, Osaka University, Suita, Osaka, Japan. ${ }^{3}$ Prophoenix Division, Food and Life-Science Laboratory, Idea Consultants, Inc., Osaka-city, Osaka, Japan. ${ }^{4}$ Division of Systems Biology, Nagoya University Graduate School of Medicine, Nagoya, Aichi, Japan. ${ }^{5}$ Genome Information Research Center, Research Institute for Microbial Diseases, Osaka University, Osaka, Japan. ${ }^{6}$ Department of Gastroenterological Surgery, Graduate School of Medicine, Osaka University, Suita, Osaka, Japan. ${ }^{7}$ Department of Gastroenterological Surgery, Nagoya City University Graduate School of Medical Sciences and Medical School, Nagoya, Aichi, Japan. ${ }^{8}$ Department of Surgery, Kyushu University Beppu Hospital, Beppu, Oita, Japan.

${ }^{9}$ Division of Molecular and Cellular Medicine, National Cancer Center Research Institute, Tokyo, Japan. ${ }^{10}$ These authors contributed equally: Masamitsu Konno, Jun Koseki, Ayumu Asai, Akira Yamagata. Correspondence and requests for materials should be addressed to K.O. (email: oof21443@ideacon.co.jp) or to M.M. (email: mmori@gesurg.med.osaka-u.ac.jp) or to H.I. (email: hishii@gesurg.med.osaka-u.ac.jp)
} 
$\mathrm{M}$ icro (mi)RNAs are broadly conserved small RNA families that are implicated in a wide variety of pathological processes, including cancer initiation and progression $^{1,2}$. Their aberrant expression in cancer tissues and remarkable stability in body fluids makes miRNAs a useful biomarker for cancer diagnosis ${ }^{3}$. However, miRNA research has traditionally employed RNA expression level to evaluate their biological significance ${ }^{4}$, and the possibility of functional heterogeneity has not been extensively investigated. In this study, we demonstrate the value of using miRNA methylation rather than expression level for cancer diagnosis.

Recent evidence suggests that a substantial fraction of mRNAs and non-coding RNAs undergo chemical modification, which is critical for embryonic development and maintenance of physical states $^{5-8}$; in fact, tRNAs-a distinct class of non-coding RNAshave a variety of RNA modifications that maintain their stability and proper functioning 9,10 . Interestingly, mature tRNAs are 70-90 nucleotides long, which is similar to the length of most miRNA precursors ${ }^{11}$. This led us to hypothesize that mature miRNAs may also undergo RNA modification. Conventional methylation detection methods such as bisulfite sequencing and methylated RNA immunoprecipitation followed by RNA sequencing (RIP-Seq) can only detect pre-established RNA modifications $^{8,12}$. In this study, we used a non-targeted mass spectrometry sequencing technique that enabled unbiased detection of RNA modifications, leading to the identification of novel methylated cytosines and adenines in several mature miRNAs in cancer cell lines, human tissues and serum.

\section{Results}

RNA methyltransferases were upregulated in gastrointestinal cancer. To investigate whether the methylated RNAs are up- or downregulated in cancer cells, we first examined the expression levels of RNA-methylation enzymes; writer proteins such as methyltransferase-like (METTL)3 and METTL14, which are methylation enzymes for adenine $\mathrm{e}^{13-15}$ and NOP2/Sun RNA methyltransferase family member (NSUN)2, which is a methylation enzyme for cytosine ${ }^{16}$. Although the expression levels were similar between gastrointestinal cancer and normal tissues, the data showed different distribution between gastrointestinal cancer and normal tissues. The expression levels tended to be upregulated in gastrointestinal cancer. (Fig. 1a, b and Supplementary Figs. 1 and 2). Moreover, the expression levels of METTL3 and the adenine demethylation enzyme AlkB homolog (ALKBH)5 were negatively correlated (Fig. 1c), suggesting that RNA methylation tended to increase in gastrointestinal cancer. To quantify the methylated miRNA in gastrointestinal cancer cells, we carried out liquid chromatography-mass spectrometry (MS) analysis $^{17}$ of small RNA fractions of three different cell lines that were size-fractionated from total RNA by ultrafiltration. RNA methyl marks including 5-methylcytosine (5mC), N6methyladenosine (m6A), 3-methylcytosine, and N1methyladenine $(\mathrm{m} 1 \mathrm{~A})$ were detected in $1-8 \%$ of total adenines and cytosines analyzed (See Supplementary Fig. 3). Importantly, the fraction of methylated miRNAs increased upon stimulation with epidermal growth factor in all cell lines examined, suggesting the existence of a systemic regulatory mechanism for RNA modification.

Methylated microRNAs have altered target inhibitory effects. To clarify the biological significance of mature miRNA methylation, we synthesized miR-200c oligonucleotides with m6A or m5C modifications at all adenines and cytosines, respectively. These oligonucleotides with or without methylation were transfected into the DICER exon 5-disrupted colorectal cancer cell line
HCT116 (HCT116 ${ }^{\mathrm{EX} 5}$ ), which has very low expression levels of endogenous miRNAs ${ }^{18}$. Gene expression profiling revealed that m6A-modified miR-200c-3p did not reduce target mRNA expression level as compared to m5C-modified or nonmethylated miR-200c-3p (See Supplementary Fig. 4).

New-method for detect the methylated microRNAs using MALDI-TOF-MS. Based on our observation that RNA methyltransferases were increased in gastrointestinal cancer cells, we speculated that methylated miRNAs could serve as biomarkers for gastrointestinal cancer. To be find the commonly methylated miRNAs in gastrointestinal cancer, we tried to m6A RIP-Seq analysis of four pancreatic cancer cell lines and identified 63 commonly-methylated miRNAs (Fig. 1d and See Supplementary Table 1). Since conventional RNA-Seq with a next-generation sequencer cannot detect methylated bases in miRNAs, we purified target miRNAs using magnetic beads with bound complementary oligonucleotides and analyzed these by matrix-assisted laser desorption/ionization time-of-flight tandem $\mathrm{MS}^{19-23}$ (Fig. 2a, b). Methylated nucleotides were detected as a peak a $+14 \mathrm{Da}$ from predicted non-methylated peaks in the mass spectrum; the methylation site was further confirmed by derivatization of nucleotides (see Methods for details). The methylation level of each miRNA was evaluated using synthetic non-methylated (let$7 a-5 p$ and miR-17-5p) and methylated (let-7a-5p and miR-17-5p) miRNAs as the ratio between peak intensities of methylated and non-methylated nucleotides. This approach provided a highly sensitive and quantitative measurement of non-methylated and methylated miRNA oligonucleotides (Fig. 2b, See Supplementary Fig. 5 and Supplementary Table 2). We used this method to assess the methylation levels of miRNAs identified by RIP-Seq (Fig. 1d, See Supplementary Table 1) in pancreatic cancer tissue. Let-7a-5p and miR-17-5p had m6A whereas miR-200c-3p and miR-21-3p had $5 \mathrm{mC}$ modifications at specific positions in the mature sequence (Fig. 2c, d, See Supplementary Fig. 6). We next measured the methylation levels of these miRNAs in pancreatic and colorectal cancer tissues and paired normal samples and found that methylation was increased in all examined cases whereas no differences in miRNA expression level were detected by quantitative reverse transcription PCR (Fig. 3a, b, See Supplementary Figs 7-10, and Supplementary Tables 3-4). Moreover, the methylation levels of these miRNAs were higher in serum samples from pancreatic and colorectal cancer patients than in those from normal subjects (Fig. 3c, d, See Supplementary Figs. 11-14), and were lower in post- as compared to pre-surgery samples (Fig. 3e, f).

Predicting structural changes of RISC complex by methylated microRNA. To clarify the biological significance of mature miRNA methylation, we carried out molecular simulations to examine the binding between Argonaute (AGO) protein and miR-17-5p and $-200 c-3 p$ and let-7a-5p (with or without methylation) as well as structural changes in the complexes. In miR$200 \mathrm{c}-3 \mathrm{p}$, the $5 \mathrm{mC}$ modification at position 9 was close to RNA recognition bases. Although there was no obvious difference between the first six nucleotides of methylated and nonmethylated miR-200c-3p in the AGO complex, variations in the binding interaction between AGO and each miRNA were observed around the methyl groups, which enhanced van der Waals interactions with the protein and thereby diminished the surrounding space. At the same time, methyl groups of the cytosine at position 9 disrupted hydrogen bonding with Ser220 of AGO likely through steric hindrance, leading to a positional shift of the guanine at position 8 that was also caused by interaction with Arg761 of AGO (See Supplementary Fig. 15). In miR-17-5p 
a

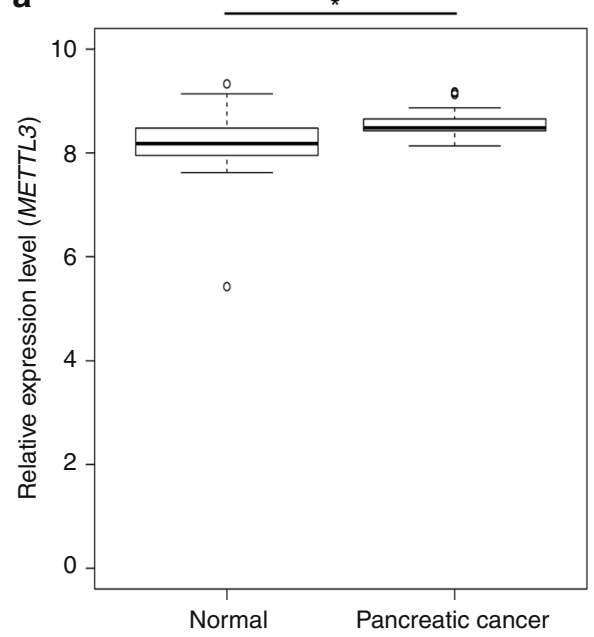

C

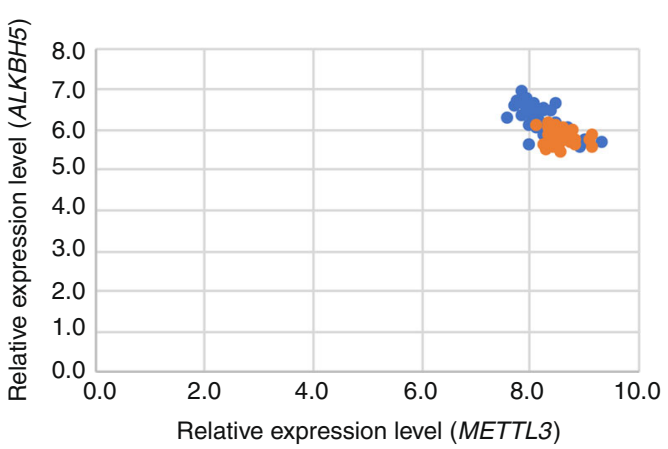

b
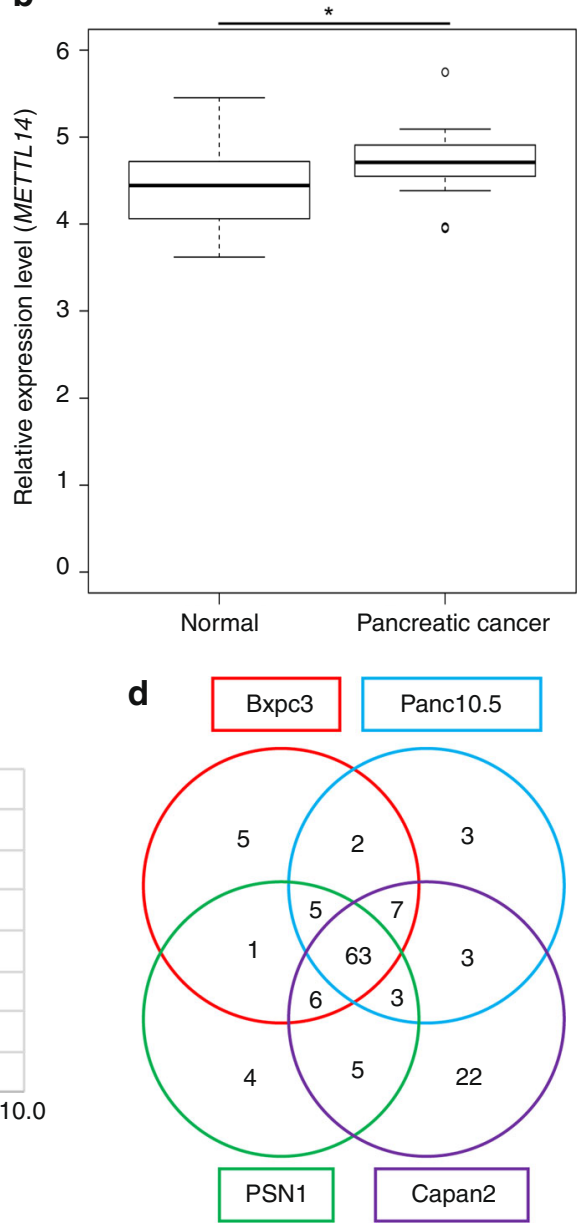

Fig. 1 Methylated miRNAs are tended to upregulated in gastrointestinal cancer. a Analysis of RNA expression levels of the RNA methylases METTL3 using a Gene Expression Omnibus (GEO) dataset (GDS4103) derived from pancreatic cancer and paired normal tissue samples from 36 patients. ${ }^{*} p=7.68 \times 10^{-5}$ (Wilcoxon's $t$ test). b Analysis of RNA expression levels of the RNA methylase METTL14 using a Gene Expression Omnibus (GEO) dataset (GDS4103) derived from pancreatic cancer and paired normal tissue samples from 36 patients. ${ }^{\star} p=0.007193$ (Wilcoxon's $t$ test). c Analysis of the correlation between METTL3 and ALKBH5 expression levels. Blue and orange points represent normal tissue and Pancreatic cancer tissue, respectively. $R$ value was -0.68 in normal tissue and -0.19 in pancreatic cancer. $\mathbf{d}$ Methylated miRNA analysis by RIP-Seq using an anti-m6A antibody. The Venn diagram shows that 63 methylated miRNAs were common to four pancreatic cancer cell lines. The box range means from the first quartile to the third quartile. The second quartile means the median of the data. The lower limit of the bar was estimated by "the first quartile $-1.5 \times$ interquartile range", and the upper limit of the bar was estimated by "the third quartile $+1.5 \times$ interquartile range"

and let-7a-5p, methylated adenines were located away from the RNA-binding site; however, m6A modification causes a large structural change in the whole complex, including around the RNA recognition site, which affects the target RNA recognition efficiency (See Supplementary Figs. 16 and 17). These findings indicate that $\mathrm{m} 6 \mathrm{~A}$ modification reduces the ability of miRNAs to suppress target mRNA translation.

Methylated microRNAs become biomarkers of gastrointestinal cancer. To evaluate the potential of miRNA methylation as a biomarker for early cancer diagnosis, we examined miRNA methylation levels in serum samples from pancreatic cancer patients and healthy controls. Methylated miR-17-5p was detected in all pancreatic cancer patient samples but was either absent or present only at a low level in controls (Fig. 4a). Moreover, miRNA methylation showed better performance in detecting early-stage pancreatic cancer than established biomarkers such as carbohydrate antigen 19-9 (CA19-9) and carcinoembryonic antigen $(\mathrm{CEA})^{24}$ (Fig. 4b, c, See Supplementary Fig. 18, and
Supplementary Table 5). Thus, evaluating miRNA methylation and not simply the expression level is a promising diagnostic strategy. To evaluate the methylation of miRNAs as a biomarker, we agree that a relatively large scale study with cancer patients and healthy controls would be necessary for the clinical use. Although the results of this study provide evidence for the biological significance of RNA methylation status in gastrointestinal cancer. Although high-throughput nucleic acid sequencing is currently the gold standard for transcriptome-level analyses, MS enables high-resolution profiling of these chemical modifications, which can aid in the early diagnosis and treatment of cancer. Moreover, elucidating the mechanisms by which methylation regulates miRNA function in the initiation and progression of cancer can lead to the development targeted therapies that can improve patient outcome.

\section{Methods}

Cell lines and culture. BxPC3, Panc10.5, PSN1, and Capan2 human pancreatic cancer cell lines were obtained from American Type Culture Collection (Manassas, VA, USA). HCT116EX5 cells were a gift from Bert Vogelstein (Johns Hopkins 
a

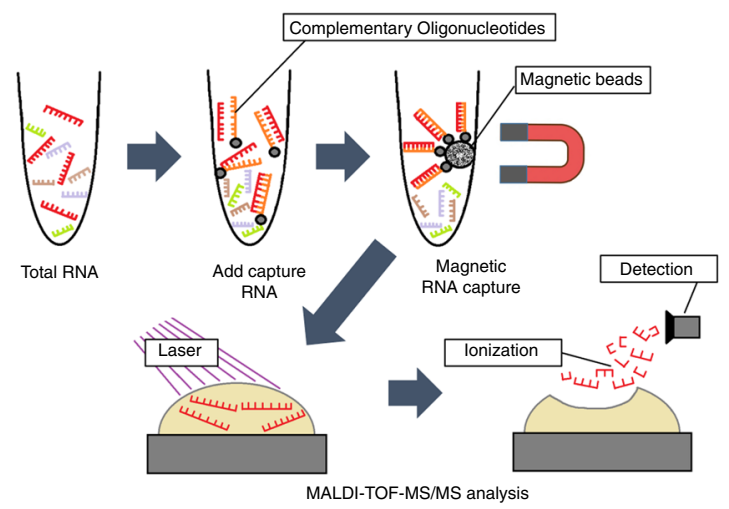

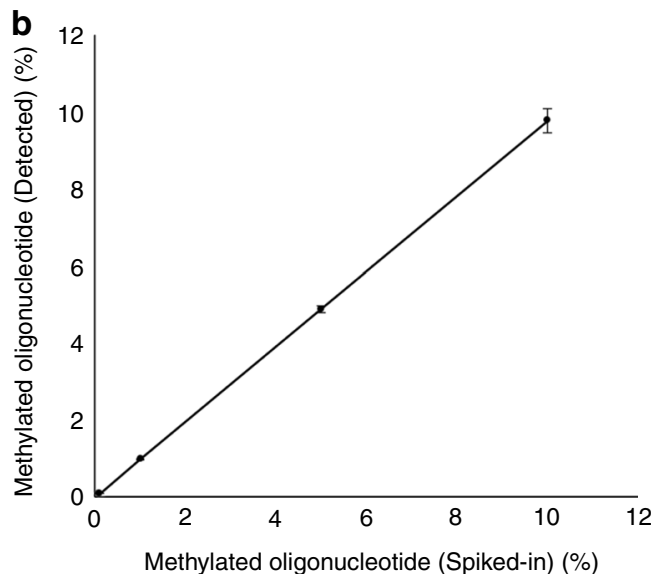

Methylated oligonucleotide (Spiked-in) (\%)

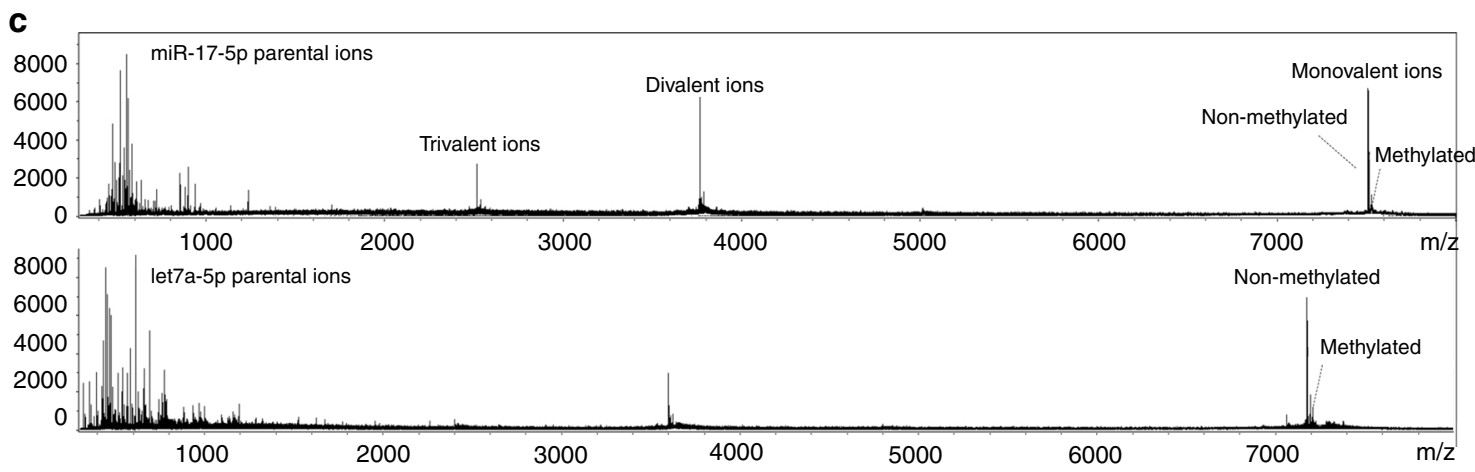

d

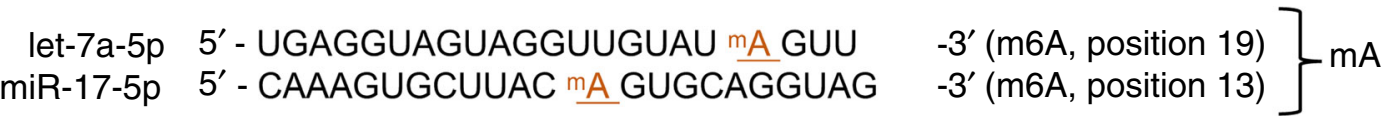

Fig. 2 Detection of methylated bases in mature miRNAs. a Schematic depiction of the procedure for detecting RNA modifications in mature miRNA sequences. Total small RNA extracted from cells was hybridized with oligonucleotides complementary to target miRNAs on magnetic beads. Captured miRNAs were eluted and applied to sample plates and then analyzed by matrix-assisted laser desorption/ionization time-of-flight tandem MS (MALDITOF-MS/MS). b Dynamic range of methylated miRNA detection. Synthetic miR-200c-3p oligonucleotides with or without methylation were mixed at the indicated concentrations and analyzed by MALDI-TOF-MS/MS. c Mass spectrum of miR-17-5p and let-7a-5p obtained from pancreatic cancer patientderived tissue. The spectrum shows monovalent, divalent, and trivalent methylated miR-17-5p RNA peaks and monovalent and divalent let-7a-5p RNA peaks (see Methods for details). d Position of methylated nucleoside in each miRNA

University, Baltimore, MS, USA). Mycoplasma testing was performed using the MycoAlert Mycoplasma Detection Kit (Lonza, Allendale, NJ). Mycoplasma testing confirmed negative results. Cells were cultured in Roswell Park Memorial Institute1640 medium supplemented with $10 \%$ fetal bovine serum (FBS) (Thermo Fisher Scientific, Waltham, MA, USA) at $37^{\circ} \mathrm{C}$ in a humidified incubator of $5 \% \mathrm{CO}_{2}$.

miRNA immunoprecipitation. miRNA immunoprecipitation. Total RNA was extracted from cells and tissues using TRIzol reagent (Thermo Fisher Scientific) according to the manufacturer's instructions. Small RNA fraction $(<100 \mathrm{nt})$ including miRNA were separated from total RNA using High Pure miRNA Isolation Kit (Roche, Basel, Switzerland) according to the manufacturer's instructions. Small RNA immunoprecipitation was performed using anti-m6A antibody (200 microug/ml) (cat. 202003 , Synaptic Systems, Goettingen, Germany) at $4^{\circ} \mathrm{C}$ for $2 \mathrm{~h}$. The miRNA-anti-m6A-antibody complex was incubated with Dynabeads Protein G (Thermo Fisher Scientific) at $4{ }^{\circ} \mathrm{C}$ for $2 \mathrm{~h}$. The mixture was obtained by magnetic separation. M6A containing microRNA was eluted from the mixture using 6.7 mM N6-Methyladenosine 5-monophosphate sodium salt (Sigma-aldrich, St. Louis, MO, USA) at $4^{\circ} \mathrm{C}$ for $2 \mathrm{~h}$

RNA microarray. After assessment for quality, $500 \mathrm{ng}$ of the extracted totalRNA was labeled with Cyanine-3 (Cy3) using the Low Input Quick Amp Labeling Kit (Agilent Technologies, Santa Clara, CA, USA). Dye incorporation and cRNA yield were assessed using the NanoDrop ND-2000 Spectrophotometer. The labeled RNAs were hybridized onto Human Oligo chip 25k (TORAY, Tokyo, Japan) for $17 \mathrm{~h}$ at $65^{\circ} \mathrm{C}$ in a rotating. After hybridization, microarrays were stringently washed for $1 \mathrm{~min}$ at room temperature with GE Wash Buffer 1 (Agilent Technologies) followed by GE Wash buffer 2 for $1 \mathrm{~min}$ at $37^{\circ} \mathrm{C}$ (Agilent Technologies) and then immediately dried by brief centrifugation. The fluorescent signals were then scanned with the Agilent DNA Microarray Scanner. The data have been submitted to the Gene Expression Omnibus database of National Center for Biotechnology Information (accession no. GSE134794).

miRNA sequencing. miRNA libraries were constructed following manufacturer instructions using the NEBNext Multiplex Small RNA Library Prep Set for Illumina (New England Biolabs, Ipswich, MA, USA) and sequenced by the HiSeq 2500 platform (Illumina, San Diego, CA, USA). After removing 3' adapters with the FASTX-toolkit, reads were aligned to the mouse and human reference genomes and transcriptomes using TopHat2 (https://ccb.jhu.edu/software/tophat/index.shtml). The human (hg38) reference sequences used for this purpose were downloaded from the University of California at Santa Cruz genome browser. We calculated the reads per kilobase of exon per million reads mapped reads using Cufflinks (http://cole-trapnell-lab.github.io/cufflinks/). Raw reads from these samples have been submitted to the Gene Expression Omnibus database of National Center for Biotechnology Information (accession no. GSE119014).

miRNA capture. Total RNA was extracted from cells and tissues using TRIzol reagent (Thermo Fisher Scientific) according to the manufacturer's instructions and annealed to single-strand DNA oligonucleotides complementary to target miRNAs that were adenine-methylated at the $5^{\prime}$ end via a C6 linker. The mixture was heated to $95^{\circ} \mathrm{C}$, then gradually cooled to $30^{\circ} \mathrm{C}$. The miRNA-DNA complex was incubated with Dynabeads M-270 Amine (Thermo Fisher Scientific) at $4^{\circ} \mathrm{C}$ for $1 \mathrm{~h}$. The mixture was heat-eluted and the supernatant was obtained by magnetic separation. Lyophilized samples were used for subsequent experiments. 
a

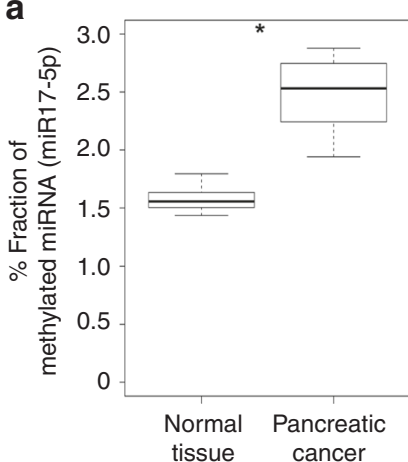

C

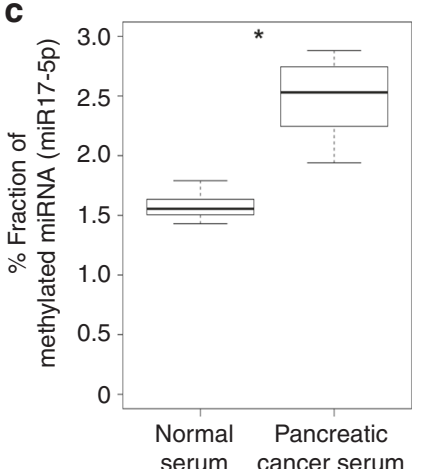

e

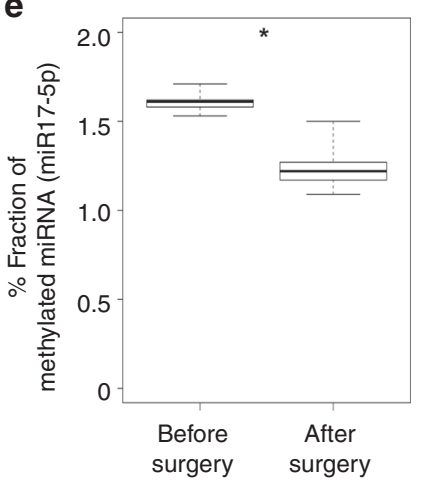

b

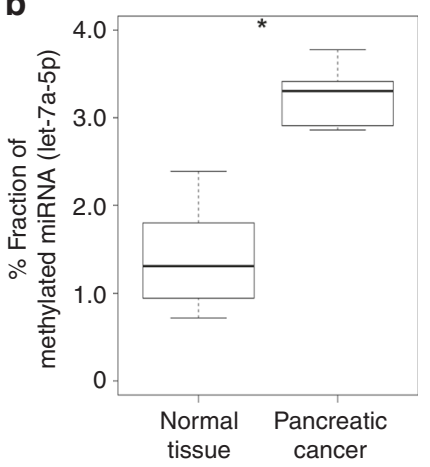

d

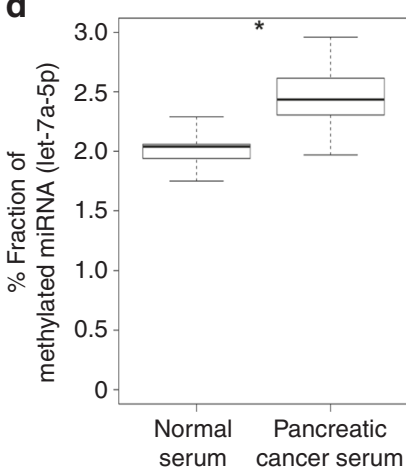

f

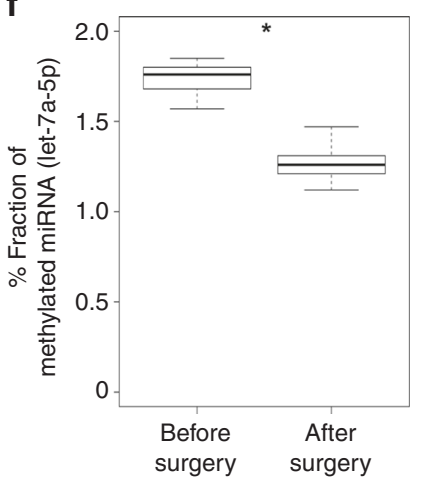

Fig. 3 Increased miRNA methylation levels in pancreatic cancer tissue and serum. $\mathbf{a}, \mathbf{b}$ Enhancement of miR-17-5p (a) and let-7a-5p (b) methylation in pancreatic cancer tissue $(n=12)$ relative to paired healthy tissue $(n=12)$. ${ }^{\star} p<0.01$ (t test). c, $\mathbf{d}$ Fraction of methylated miRNA at a specific position of miR-17-5p (c) and let-7a-5p (d) in serum derived from pancreatic cancer patients $(n=5)$ and healthy controls $(n=5)$. Healthy control serum was obtained from liver transplantation donors who were confirmed as having no cancer by endoscopy, computed tomography, and by detection of several tumor markers. ${ }^{*} p<0.01$ ( $t$ test). e, $\mathbf{f}$ Fraction of methylated miRNA at a specific position of miR-17-5p (e) and let-7a-5p (f) in serum derived from pancreatic cancer patients before $(n=21)$ and after $(n=21)$ surgery. Healthy control serum was obtained from liver transplantation donors who were confirmed as having no cancer by endoscopy, computed tomography, and by detection of several tumor markers. ${ }^{*} p<0.01$ ( $t$ test). The box range means from the first quartile to the third quartile. The second quartile means the median of the data. The lower limit of the bar was estimated by "the first quartile $-1.5 \times$ interquartile range", and the upper limit of the bar was estimated by "the third quartile $+1.5 \times$ interquartile range

Matrix-assisted laser desorption/ionization time-of-flight mass spectrometry (MALDI-TOF-MS). Captured miRNAs were purified with a Zip Tip C18 column (Millipore, Billerica, MA, USA) according to the manufacturer's protocol. Purified samples were mixed with an aqueous solution of 3-hydroxypicolinic acid (Bruker

Daltonics, Bremen, Germany) at a ratio of 1:1 (v/v) and applied to the target plate. One microliter of the mixture was applied to an MTP AnchorChip 384 target plate (Bruker Daltonics) and air-dried at room temperature. MALDI-TOF-MS analysis was performed with an ultrafleXtreme MALDI-TOF/TOF mass spectrometer (Bruker Daltonics) operated in negative ion and reflectron modes. Spectra were manually acquired using FlexControl software (v.3.3.108.0) (Bruker Daltonics). An analysis of the RNAs following hydrazine treatment revealed that the peaks contained $\mathrm{m} 5 \mathrm{C}^{25}$. Methylated adenines were determined to be $6 \mathrm{~mA}$ by sequential MS analysis following treatment with dimethylsulfate, which preferentially alkylates the $\mathrm{N} 1$ of adenines in $\mathrm{RNA}^{26}$.

Epidermal growth factor (EGF) stimulation. Cells were seeded in 6-well plates $(2 \times 105$ cells/well $)$ and incubated with medium containing $10 \%$ FBS. After $48 \mathrm{~h}$, the medium was replaced with one containing $1 \%$ FBS. After another $24 \mathrm{~h}$, recombinant EGF (Sigma-Aldrich) was added at a concentration of $100 \mathrm{ng} / \mathrm{ml}$; untreated cells served as the control group. The cells were collected $12 \mathrm{~h}$ later for analysis.

Liquid chromatography-tandem MS (LC-MS/MS). Total RNA was purified by ultrafiltration using an Eclipse XDB-C18 high-performance LC column (Agilent Technologies) with a molecular weight cut-off of $3 \mathrm{kDa}$. Isolated small RNA fractions were hydrolyzed into nucleosides in a reaction mixture containing benzonase, phosphodiestrase I, and alkaline phosphatase. LC-MS/MS analysis was performed on an Agilent 6400 mass spectrometer (Agilent Technologies).

Transfection of synthetic oligonucleotides. Double-stranded RNA oligonucleotides with or without methyl groups were synthesized by Gene Design (Osaka, Japan). The sequence of non-methylated and methylated nucleosides was verified by MALDI-TOF-MS/MS. miRNA sequences were obtained from miRBASE (release 21; http://www.mirbase.org/). The sequence of each oligonucleotide is shown in Fig. 2d. Synthetic miRNAs were transfected into cells using Lipofectamine 3000 (Invitrogen) according to the manufacturer's protocol.

Expression data analysis. In order to compare the difference of gene expression changes adopted miR-200c-3p of cytosine-methylated and non-methylated forms to, Gene set enrichment analysis (GSEA) was performed using the fgsea package ${ }^{27}$ on $\mathrm{R}$ version $3.5 .225^{28}$. In these analyses, we used the original gene set for targets of above micro RNAs based on Tarbase (http://diana.imis.athena-innovation.gr/ DianaTools/index.php? $r=$ tarbase/index $)^{29}$, which was decided based on some experimental facts. GSEA was performed on the ranked list for changes between two experimental conditions (non-methylated or methylated micro RNA and control) on gene sets of these target genes.

Real-time quantitative reverse transcription polymerase chain reaction (qRTPCR). The TaqMan MicroRNA Reverse Transcription kit (Applied Biosystems, Foster City, CA, USA) was used for miRNA quantification according to the manufacturer's protocols.

Molecular dynamics simulations. To predict the binding of methylated and nonmethylated miRNAs to the human AGO2 protein (See Supplementary Figs. 15-17), we used the X-ray structure of AGO2/RNA as the guide complex (PDB ID: 4OLB 30 and $4 \mathrm{~W} 5 \mathrm{~N}^{31}$ ). Initially, the RNA-binding bases were substituted with the corresponding bases in each miRNA. For each miRNA complex structure, molecular dynamics simulations were performed under organic conditions (1 atm and $\sim 37^{\circ}$ C). After thermodynamic sampling, energy minimizations were carried out to predict the docking of the structures. All calculations were performed with AMBER99 force field ${ }^{32}$ using the Amber 12 program (http://ambermd.org/) 33 .

Clinical samples. Colorectal and gastric cancer and paired normal tissue samples (located $>5 \mathrm{~cm}$ from the malignant region) were obtained during surgery. All patients underwent resection of the primary tumor at Osaka University Hospital (Osaka, Japan) and affiliated hospitals. Blood samples from pancreatic cancer patients were collected before surgery. Healthy controls had no visible lesions by diagnostic imaging and no abnormal findings in the blood examination. Informed consent was obtained from all participants. We have complied with all relevant ethical regulations for work with human participants. The study protocols were approved by the ethics committee of Osaka University (approval nos. 663, 15222-2, and 08226-5).

Reporting summary. Further information on research design is available in the Nature Research Reporting Summary linked to this article.

\section{Data availability}

All the data supporting the findings of this study are available within the article and its supplementary information files and from the corresponding author upon reasonable request. Sequence data for Fig. 1d have been deposited in the NCBI GEO data set with 
a

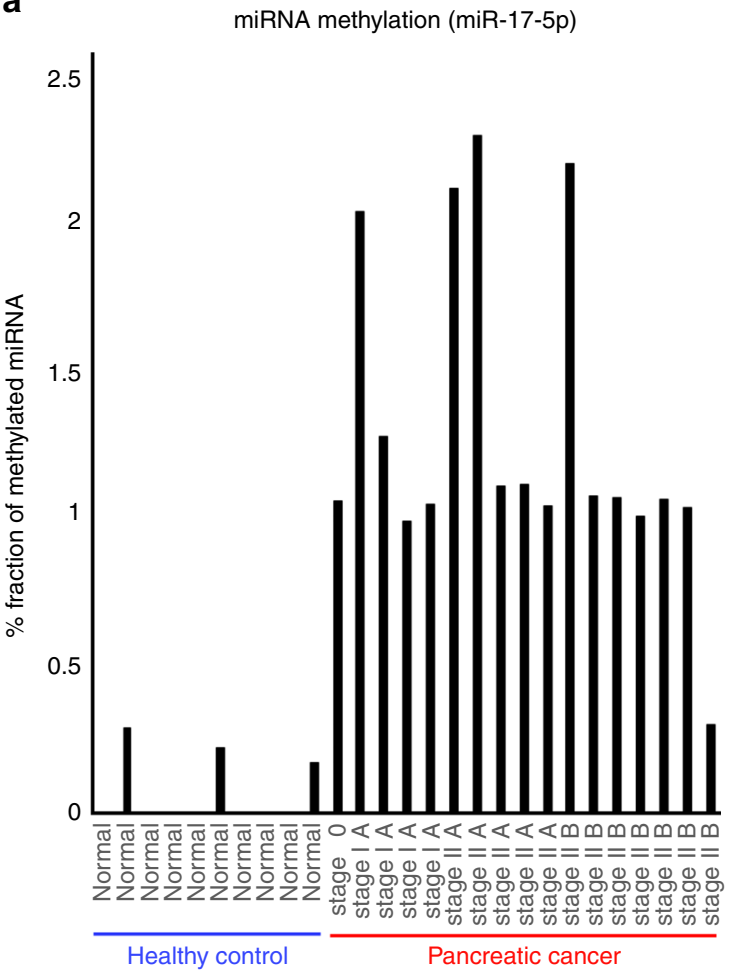

b

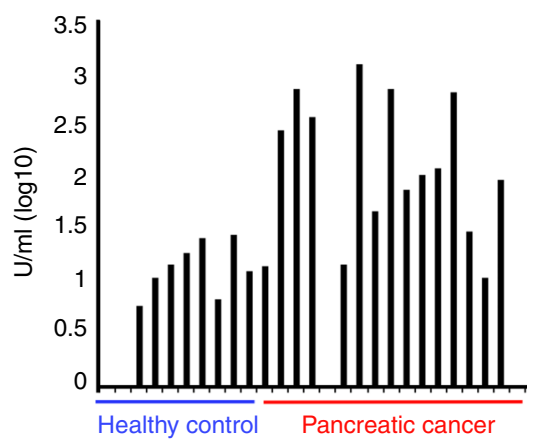

C

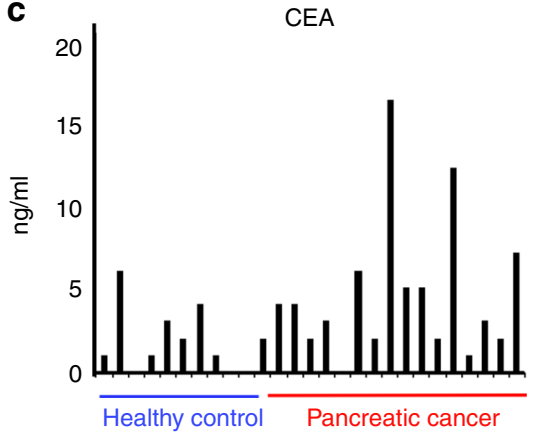

Fig. 4 Diagnostic value of methylated miRNA in early-stage pancreatic cancer. a Left, bar graph showing the fraction of methylated miRNA of miR-17-5p in serum from pancreatic cancer patients $(n=17)$ and healthy controls $(n=10)$. The clinical stages of pancreatic cancer are indicated at the bottom. Expression below the detection limit is shown as zero. Right, quantitative analysis of carbohydrate antigen (CA19-9) (b) and carcinoembryonic antigen (CEA) (c) expression in the same set of samples. See Supplementary Table 5 for detailed clinicopathological information of each patient

accession code GSE119014; Microarray data for Supplementary Fig. 4 have been deposited in the NCBI GEO data set with accession code GSE134794.

Public datasets used in this study can be found in GEO under the accession numbers: GDS4103 (https://www.ncbi.nlm.nih.gov/geo/query/acc.cgi?acc=GSE15471), and GDS4382 (https://www.ncbi.nlm.nih.gov/geo/query/acc.cgi?acc=GSE32323). X-ray structure used in this study can be found in PDB ID: 4OLB and 4W5N2 (http://www. rcsb.org/structure/4W5N).

Received: 27 September 2016 Accepted: 6 August 2019 Published online: 29 August 2019

\section{References}

1. Nicoloso, M. S., Spizzo, R., Shimizu, M., Rossi, S. \& Calin, G. A. MicroRNAs-the micro steering wheel of tumour metastases. Nat. Rev. Cancer 9, 293-302 (2009).

2. Schetter, A. J. et al. MicroRNA expression profiles associated with prognosis and therapeutic outcome in colon adenocarcinoma. JAMA 299, 425-436 (2008).

3. Schwarzenbach, H., Nishida, N., Calin, Ga \& Pantel, K. Clinical relevance of circulating cell-free microRNAs in cancer. Nat. Rev. Clin. Oncol. 11, 145-156 (2014).

4. Nair, V. S., Maeda, L. S. \& Ioannidis, J. P. Clinical outcome prediction by microRNAs in human cancer: a systematic review. J. Natl. Cancer Inst. 104, 528-540 (2012).

5. Dominissini, D. et al. Topology of the human and mouse m6A RNA methylomes revealed by m6A-seq. Nature 485, 201-206 (2012).

6. Wang, Y. et al. N6-methyladenosine modification destabilizes developmental regulators in embryonic stem cells. Nat. Cell Biol. 16, 191-198 (2014).

7. Wang, X. et al. N6-methyladenosine-dependent regulation of messenger RNA stability. Nature 505, 117-120 (2014).

8. Meyer, K. D. \& Jaffrey, S. R. The dynamic epitranscriptome: N(6)methyladenosine and gene expression control. Nat. Rev. Mol. Cell Biol. 15, 313-326 (2014).

9. Thompson, D. M. \& Parker, R. Stressing out over tRNA cleavage. Cell 138, 215-219 (2009).
10. Torres, A. G., Batlle, E. \& Ribas de Pouplana, L. Role of tRNA modifications in human diseases. Trend Mol. Med. 20, 306-314 (2014).

11. Krol, J., Loedige, I. \& Filipowicz, W. The widespread regulation of microRNA biogenesis, function and decay. Nat. Rev. Genet. 11, 597-610 (2010).

12. Squires, J. E. et al. Widespread occurrence of 5-methylcytosine in human coding and non-coding RNA. Nucl. Acid. Res. 40, 5023-5033 (2012).

13. Masamitsu Konno, Masateru Taniguchi, Hideshi Ishii. Significant Epitranscriptomes in Heterogeneous Cancer. Cancer Science 110, 2318-2327 (2019).

14. Bokar J. A. et. al. Purification and cDNA cloning of the AdoMet-binding subunit of the human mRNA (N6-adenosine)-methyltransferase. RNA 3, 1233-47 (1997).

15. Jianzhao Liu, Yanan Yue, et al. A METTL3-METTL14 complex mediates mammalian nuclear RNA N6-adenosine methylation. Nature Chemical Biology 10, 93-95 (2014).

16. Michaela Frye, Fiona M. Watt. The RNA Methyltransferase Misu (NSun2) Mediates Myc-Induced Proliferation and Is Upregulated in Tumors. Current Biology 16, 971-981 (2006)

17. Gaston, K. W. \& Limbach, P. A. The identification and characterization of non-coding and coding RNAs and their modified nucleosides by mass spectrometry. RNA Biol. 11, 1568-1585 (2014).

18. Cummins, J. M. et al. The colorectal microRNAome. Proc. Natl Acad. Sci. USA 103, 3687-3692 (2006)

19. Bray, M. S., Boerwinkle, E. \& Doris, P. A. High-throughput multiplex SNP genotyping with MALDI-TOF mass spectrometry: practice, problems and promise. Hum. Mutat. 17, 296-304 (2001).

20. Kirpekar, F. et al. Matrix assisted laser desorption/ionization mass spectrometry of enzymatically synthesized RNA up to $150 \mathrm{kDa}$. Nucl. Acid. Res. 22, 3866-3870 (1994).

21. Kirpekar, F., Douthwaite, S. \& Roepstorff, P. Mapping posttranscriptional modifications in $5 \mathrm{~S}$ ribosomal RNA by MALDI mass spectrometry. RNA $\mathbf{6}$, 296-306 (2000).

22. Zhu, L., Parr, G. R., Fitzgerald, M. C., Nelson, C. M. \& Smith, L. M. Oligodeoxynucleotide fragmentation in MALDI/TOF mass spectrometry using 355-Nm radiation. J. Am. Chem. Soc. 117, 6048-6056 (1995).

23. Mengel-Jørgensen, J. \& Kirpekar, F. Detection of pseudouridine and other modifications in tRNA by cyanoethylation and MALDI mass spectrometry. Nucl. Acid. Res. 30, e135 (2002). 
24. Hanash, S. M., Baik, C. S. \& Kallioniemi, O. Emerging molecular biomarkers-blood-based strategies to detect and monitor cancer. Nat. Rev. Clin. Oncol. 8, 142-150 (2011).

25. Behm-Ansmant, I., Helm, M. \& Motorin, Y. Use of specific chemical reagents for detection of modified nucleotides in RNA. J. Nucl. Acid. 2011, 1-17 (2011).

26. $\mathrm{Su}, \mathrm{D}$. et al. Quantitative analysis of ribonucleoside modifications in tRNA by HPLC-coupled mass spectrometry. Nat. Protoc. 9, 828-841 (2014).

27. Sergushichev, A., An algorithm for fast preranked gene set enrichment analysis using cumulative statistic calculation, Cold Spring Harbor Lab J. (2016). (http://biorxiv.org/content/early/2016/06/20/060012)

28. R. Core Team. R: A language and environment for statistical computing. $R$ Foundat. Statist. Comput., (Vienna, Austria, 2014). (http://www.R-project.org/)

29. Grimson, A. et al. MicroRNA targeting specificity in mammals: determinants beyond seed pairing. Mol. Cell 27, 91-105 (2007).

30. Schirle, N. T. \& MacRae, I. J. The crystal structure of human Argonaute2. Science 336, 1037-1040 (2012).

31. Reed, A. E., Curtiss, L. A. \& Weinhold, F. Intermolecular interactions from a natural bond orbital, donor-acceptor viewpoint. Chem. Rev. 88, 899-926 (1988)

32. Wang, J. et al. How well does a restrained electrostatic potential (RESP) model perform in calculating conformational energies of organic and biological molecules? J. Comp. Chem. 21, 1049-1074 (2000).

33. Case, D. A. et al. AMBER 12 12th edn. (University of California, San Francisco, 2012).

\section{Acknowledgements}

We thank B. Vogelstein, Johns Hopkins University, USA, for providing DICER exon 5-disrupted cell lines; A. Inoue, K. Asukai, K. Yanagisawa, R. Toshiyama, K. Matsushita, Y. Ueda and N. Nishida, Osaka University, Japan, for collecting clinical samples; M Ozaki and Y. Noguchi, Osaka University, for technical assistance; and Osamu Nureki, University Tokyo, for useful discussions. This work was supported in part by a Grant-inAid for Scientific Research from the Ministry of Education, Culture, Sports, Science and Technology (15H05791; 17H04282; 17K19698; 18K16356; 18K16355); AMED, Japan $(16 \mathrm{~cm} 0106414 \mathrm{~h} 0001 ; 17 \mathrm{~cm} 0106414 \mathrm{~h} 0002)$. Partial support was received from Takeda Science Foundation, Senri Life Science Foundation, Osaka Cancer Society, Princess Takamatsu Cancer Research Fund, Yasuda Medical, Pancreas Research Foundation, Nakatani Foundation, and Nakatomi Foundation of Japan.

\section{Author contributions}

H.I. initiated the study. M.K., J.K., A.A., T.S., Y.D., M.M. and H.I. designed the experiments and wrote the manuscript. M.K., J.K., A.A., A.Y., T.S., D.M., D.O., K.K., T.O. and K.O. performed in vitro experiments, data analysis, and mass spectrometry analysis. J.K. performed in silico molecular simulations. T.M., H.E., S.T., T.S., K.M., Y.D. and M. M. provided clinical samples.

\section{Additional information}

Supplementary Information accompanies this paper at https://doi.org/10.1038/s41467019-11826-1.

Conflict of interest: M.K. and T.S. have the following financial interests: Chugai Co., Ltd.; Yakult Honsha Co., Ltd.; Ono Pharmaceutic Co., Ltd. (Osaka, Japan); and Merck Co., Ltd (Tokyo, Japan). H.I. and J.K. have the following financial interests: partial institutional endowments were received from Taiho Pharmaceutical Co., Ltd.; Kinshu-kai Medical Corporation (Osaka, Japan); Unitech Co. Ltd. (Chiba, Japan); IDEA Consultants Inc. (Tokyo, Japan). A.Y., and K.O. are employed in IDEA Consultants Inc.

(Tokyo, Japan). All the remaining authors declare no competing interests.

Reprints and permission information is available online at http://npg.nature.com/ reprintsandpermissions/

Peer review information: Nature Communications thanks the anonymous reviewers for their contribution to the peer review of this work.

Publisher's note: Springer Nature remains neutral with regard to jurisdictional claims in published maps and institutional affiliations.

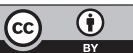

Open Access This article is licensed under a Creative Commons Attribution 4.0 International License, which permits use, sharing, adaptation, distribution and reproduction in any medium or format, as long as you give appropriate credit to the original author(s) and the source, provide a link to the Creative Commons license, and indicate if changes were made. The images or other third party material in this article are included in the article's Creative Commons license, unless indicated otherwise in a credit line to the material. If material is not included in the article's Creative Commons license and your intended use is not permitted by statutory regulation or exceeds the permitted use, you will need to obtain permission directly from the copyright holder. To view a copy of this license, visit http://creativecommons.org/ licenses/by/4.0/.

(C) The Author(s) 2019 